\title{
无容器制备 $\mathrm{Er}^{3+} / \mathrm{Yb}^{3+}$ 共掺 $\mathrm{La}_{2} \mathrm{O}_{3}-\mathrm{TiO}_{2}-\mathrm{ZrO}_{2}$ 玻璃及其 上转换发光研究
}

朱美娟 ${ }^{1}$, 余建定 ${ }^{2}$, 张明辉 ${ }^{2}$, 谷彦静 ${ }^{2}$, 李 勤 $^{2}$, 方必军 ${ }^{1}$, 赵洪阳 ${ }^{2}$, 沈 清 ${ }^{3}$ (1. 常州大学 材料科学与工程学院, 常州 213164; 2. 中国科学院 上海硅酸盐研究所, 上海 200050; 3. 常州宇环光 电有限公司, 常州 213164)

摘 要: 利用无容器技术制备了 $\left(\mathrm{La}_{0.94-x} \mathrm{Er}_{0.06} \mathrm{Yb}_{x}\right)\left(\mathrm{Ti}_{0.95} \mathrm{Zr}_{0.05}\right)_{2.25} \mathrm{O}_{6}(x=0 \sim 0.24$, 间隔 0.04)球状透明玻璃, 其稀土离子 掺杂浓度最大值达到 $30 \%$ 。通过 DTA 分析发现, 玻璃具有很好的热稳定性, $x=0$ 时玻璃化转变温度 $T_{\mathrm{g}}$ 和析晶起始温 度 $T_{0}$ 分别为 $818^{\circ} \mathrm{C}$ 和 $906^{\circ} \mathrm{C}, \Delta T\left(\Delta T=T_{0}-T_{\mathrm{g}}\right)$ 为 $88^{\circ} \mathrm{C}$, 玻璃形成能力较低。随着 $\mathrm{Yb}^{3+}$ 浓度提高, $T_{\mathrm{g}} 、 T_{\mathrm{o}}$ 和 $\Delta T$ 逐渐下降, 说 明 $\mathrm{Yb}^{3+}$ 降低了玻璃的热稳定性和形成能力。利用紫外可见分光光度计测定了样品的吸收/透过光谱, 玻璃在 $975 \mathrm{~nm}$ 具 有很强的吸收峰, 表明 $\mathrm{Yb}^{3+}$ 可以有效提高玻璃对入射光的吸收强度; 在可见光范围内除特征吸收外具有近 $70 \%$ 的透 过率, 说明玻璃具有良好的透可见光性能, 有望获得高强上转换发光输出。上转换苂光光谱研究表明: 在 $980 \mathrm{~nm}$ 激光 泵浦下, 获得了中心位于 535、554 和 $672 \mathrm{~nm}$ 处的绿、红发光带, $x=0.16$ 的发光最强, $672 \mathrm{~nm}$ 处的红光强度是 $x=0$ 的 近 130 倍。上转换发光强度与洜浦功率关系的分析表明: $535 、 554 \mathrm{~nm}$ 处的绿光和 $672 \mathrm{~nm}$ 处的红光发光均是双光子 发光过程。

关 键 词: 上转换发光; 无容器技术; 钛酸盐玻璃; $\mathrm{Er}^{3+} / \mathrm{Yb}^{3+}$ 共掺杂

中图分类号: TQ174 文献标识码: A

\section{Upconversion Luminescence of $\mathrm{Er}^{3+} / \mathrm{Yb}^{3+} \mathrm{Co}$-doped $\mathrm{La}_{2} \mathrm{O}_{3}-\mathrm{TiO}_{2}-\mathrm{ZrO}_{2}$ Glasses Prepared by Containerless Processing}

\author{
ZHU Mei-Juan ${ }^{1}$, YU Jian-Ding ${ }^{2}$, ZHANG Ming-Hui², GU Yan-Jing ${ }^{2}$, LI Qin², \\ FANG Bi-Jun ${ }^{1}$, ZHAO Hong-Yang ${ }^{2}$, SHEN Qing ${ }^{3}$
}

(1. School of Materials Science and Engineering, Changzhou University, Changzhou 213164, China; 2. Shanghai Institute of Ceramics, Chinese Academy of Sciences, Shanghai 200050, China; 3. Changzhou Yuhuan Optoelectronic Co., Ltd., Changzhou 213164, China)

\begin{abstract}
Sphere transparent titanate glasses $\left(\mathrm{La}_{0.94-x} \mathrm{Er}_{0.06} \mathrm{Yb}_{x}\right)\left(\mathrm{Ti}_{0.95} \mathrm{Zr}_{0.05}\right)_{2.25} \mathrm{O}_{6}(x=0-0.24$, in steps of 0.04$)$ were prepared by containerless processing. The DTA curve shows that the glass $(x=0)$ has high glass transition temperature $\left(T_{\mathrm{g}}=818^{\circ} \mathrm{C}\right)$ and onset temperature of crystallization $\left(T_{\mathrm{o}}=906^{\circ} \mathrm{C}\right)$. The thermal stability and the glass forming ability decrease with the $\mathrm{Yb}^{3+}$ concentration increasing. Strong absorption bands at $975 \mathrm{~nm}$ are observed, showing effective absorption of $\mathrm{Yb}^{3+}$ at $975 \mathrm{~nm}$. The transmittance is almost 70\% in 300-3000 nm except characteristic absorption of $\mathrm{Er}^{3+}$ and $\mathrm{Yb}^{3+}$. Three emission bands centered at 535, 554, $672 \mathrm{~nm}$ are obtained at the excitation of $980 \mathrm{~nm}$ laser. When the $\mathrm{Yb}^{3+}$ concentration $x$ is 0.16 , the glass sample performs the strongest upconversion luminescence,
\end{abstract}

收稿日期：2014-03-31; 收到修改稿日期：2014-09-12

基金项目: 常州市国际科技合作项目(CZ20123004); 上海硅酸盐研究所科技创新项目(Y32ZC1110G, Y22ZB1110G)

International Cooperation Project of Changzhou City(CZ20123004); Science and Technology Innovation Project in Shanghai Institute of Ceramics(Y32ZC1110G, Y22ZB1110G)

作者简介: 朱美娟(1989-), 女, 硕士研究生. E-mail:whitezmj@126.com

通讯作者: 余建定, 教授. E-mail: yujianding@mail.sic.ac.cn; 方必军, 教授. E-mail: fangbj@cczu.edu.cn 
which emission intensity at $672 \mathrm{~nm}$ is almost 130 times as high as that of $\mathrm{Er}^{3+}$-doped glass at room temperature.

Key words: upconversion luminescence; containerless processing; titanate glass; $\mathrm{Er}^{3+} / \mathrm{Yb}^{3+}$ co-doped glass

近年来, 稀土掺杂上转换玻璃引起了广泛关注, 其应用领域涉及高密度光学存储、光纤通讯、三维显 示等 ${ }^{[1-3]}$ 。 $\mathrm{Er}^{3+}$ 能级十分丰富且分布均匀, 有利于单光 束洜浦, 具有较高的猝灭浓度, 较多的泵浦途径 ${ }^{[4-5]}$, 是上转换研究最多的稀土离子。但是, $\mathrm{Er}^{3+}$ 对 $980 \mathrm{~nm}$ 原浦波长的吸收截面较小, 而 $\mathrm{Yb}^{3+}$ 在 $980 \mathrm{~nm}$ 有很强 的吸收, 所以 $\mathrm{Yb}^{3+}$ 常与 $\mathrm{Er}^{3+}$ 共掺来提高材料对泵浦激 光的吸收 ${ }^{[6-7]}$ 。

目前限制上转换发光材料应用的主要瓶颈是发 光效率不高, 而基质材料的最大声子能量是影响上 转换发光效率的主要因素。最大声子能量越小, 稀土 离子的非辐射弛豫速率越小, 上转换效率越高。具有 较低声子能量的氟化物和氟氧化物是目前上转换发 光玻璃研究的主要对象 ${ }^{[5,8-9]}$, 但是, 其机械强度、热 稳定性和耐腐蚀性较差, 很难实现实用化 ${ }^{[10]}$ 。与氟化 物相比, 氧化物玻璃具有优良的机械强度、热稳定性 和耐腐蚀性能，在工程应用领域具有更好的开发前 景。 $\mathrm{TiO}_{2}$ 是一种声子能量较低的材料 $\left(\sim 639 \mathrm{~cm}^{-1}\right)$, 且 具有很高的折射率 $(n=2.5)$ 。根据稀土发光离子的 J-O 理论 ${ }^{[11-12]}$, 玻璃的高折射率有利于提高发光离子的 辐射跃迁几率和受激发射截面, 获得较强上转换发 光。但是 $\mathrm{TiO}_{2}$ 属于玻璃中间体, 玻璃形成能力低。 利用传统熔融淬冷技术制备的二元钛酸盐玻璃 ${ }^{[13]}$, 由于制备方法的限制，只能获得薄膜或薄片状玻璃 体，无法获得块体 $\mathrm{TiO}_{2}$ 基氧化物玻璃。

无容器技术是利用磁场、电场和气体等作用力 来抵消重力而使物体悬浮, 再利用激光加热熔化进 行非平衡态凝固的过程。该技术不仅可避免外来杂 质的污染, 而且可以抑制异质形核, 使熔体获得深 过冷而实现快速凝固。因此, 无容器技术可以使玻 璃形成能力较低的材料玻璃化。Yu 等 ${ }^{[14-15]}$ 在不添加 玻璃形成体的情况下, 利用气悬浮技术制备了球形 $\mathrm{BaTi}_{2} \mathrm{O}_{5}$ 玻璃, 发现其折射率高达 2.15 , 并用该技术 制备了 $\mathrm{La}_{4} \mathrm{Ti}_{9} \mathrm{O}_{24}$ 玻璃, 进一步提高其折射率 ${ }^{[16]}$ 。 $\mathrm{Pan}^{[17]}$ 和 Zhang ${ }^{[18-19]}$ 等利用气县浮技术制备了稀土 离子掺杂的 $\mathrm{TiO}_{2}$ 基玻璃, 获得了肉眼可见的上转换 发光。本实验利用气悬浮技术制备了不同 $\mathrm{Yb}^{3+}$ 掺杂 浓度的 $\mathrm{Er}^{3+} / \mathrm{Yb}^{3+}$ 共掺 $\mathrm{La}_{2} \mathrm{O}_{3}-\mathrm{TiO}_{2}-\mathrm{ZrO}_{2}$ 玻璃 $\left(\mathrm{LTZ}: \mathrm{Er}^{3+} / \mathrm{Yb}^{3+}\right)$, 研究了 $\mathrm{Yb}^{3+}$ 浓度对玻璃形成能力、 热稳定性、吸收/透过率及上转换发光性能的影响, 探讨其上转换发光机理。

\section{1 实验方法}

\section{1 玻璃制备}

LTZ: $\mathrm{Er}^{3+} / \mathrm{Yb}^{3+}$ 玻璃的组成是 $\left(\mathrm{La}_{0.94-x} \mathrm{Er}_{0.06} \mathrm{Yb}_{x}\right)-$ $\left(\mathrm{Ti}_{0.95} \mathrm{Zr}_{0.05}\right)_{2.25} \mathrm{O}_{6}$, 其中 $x=0 、 0.04 、 0.08 、 0.12 、 0.16$ 、 0.20 和 0.24 , 分别记为样品 $1 \# 、 2 \# 、 3 \# 、 4 \# 、 5 \# 、$ $6 \# 、 7 \#$ 。按化学计量比称取相应质量的原料 $\mathrm{La}_{2} \mathrm{O}_{3}(99.99 \%)$ 、 $\mathrm{TiO}_{2}(99.99 \%) 、 \mathrm{ZrO}_{2}(99.9 \%)$ 、 $\mathrm{Er}_{2} \mathrm{O}_{3}(99.99 \%) 、 \mathrm{Yb}_{2} \mathrm{O}_{3}(99.99 \%)$ 共 $2 \mathrm{~g}$, 置于玛瑙研针 中用酒精湿磨两次。将研磨均匀的粉体在电炉中预 烧, 以 $10^{\circ} \mathrm{C} / \mathrm{min}$ 的速率升温至 $1000^{\circ} \mathrm{C}$ 保温 $10 \mathrm{~h}$ 后随 炉冷却。预烧后的粉体用 PVA 溶液造粒, 在 $15 \mathrm{MPa}$ 下压制成圆柱状材料，置于 $\mathrm{Al}_{2} \mathrm{O}_{3}$ 坩埚中在电炉中 进行烧结, 以 $10^{\circ} \mathrm{C} / \mathrm{min}$ 的速率升温至 $1200^{\circ} \mathrm{C}$ 保温 $8 \mathrm{~h}$ 后随炉冷却。随后取 $75 \mathrm{mg}$ 样品, 置于气悬浮激光加热 炉的喷嘴中, 从喷嘴底部通入 $\mathrm{O}_{2}$ 使样品悬浮, 激光从 顶部对样品进行加热使其成球形熔体。对样品进行升 温和冷却, 排除气泡, 在稳定状态下保温 $1 \mathrm{~min}$, 待样 品均一化后关闭激光器, 熔体以 $300^{\circ} \mathrm{C} / \mathrm{min}$ 的速率降 温, 快速凝固成直径 $3 \mathrm{~mm}$ 的玻璃球。双面抛光得到 厚度 $1.5 \mathrm{~mm}$ 的样品, 用于性能测试。有关气悬浮激 光加热炉的详细介绍见文献[15]。

\section{2 玻璃表征与测试}

采用 Rigaku 公司的 TG 8120 热分析仪在空气气 氛下以 $10^{\circ} \mathrm{C} / \mathrm{min}$ 升温速率对玻璃进行差热分析 (DTA)。采用 Varian 公司 Cary 5000 型紫外-可见-近 红外分光光度计测试样品在 400 1600 nm 范围的吸 收曲线和在 300 3000 nm 范围的透过曲线。利用 Edinburgh 公司 FLS 920 苂光光谱仪测试样品的上转 换荧光光谱, 以 $980 \mathrm{~nm}$ 半导体激光器作为泵浦光源, 泵浦功率为 $120 \mathrm{~mW}$, 整个测试中泵浦光源功率大小 和位置保持不变, 所有样品均放置在同一位置。改变 泵浦功率, 测量 $\mathrm{LTZ}: \mathrm{Er}^{3+} / \mathrm{Yb}^{3+}(x=0.16)$ 在不同功率下 的上转换荧光光谱。以上测试均在常温下进行。

\section{2 结果与讨论}

\section{1 无容器制备 $\mathrm{LTZ}: \mathrm{Er}^{3+} / \mathrm{Yb}^{3+}$ 玻璃}

图1简单给出了利用无容器技术制备LTZ: $\mathrm{Er}^{3+} / \mathrm{Yb}^{3+}$ 玻璃的工艺步骤。气悬浮技术可以快速地制备玻璃, 
成型原料经过激光加热熔化, 最后快速凝固成玻璃 球, 整个过程仅需 $2 \mathrm{~min}$ 。玻璃的组成为 $\left(\mathrm{La}_{0.94-\mathrm{x}} \mathrm{Er}_{0.06} \mathrm{Yb}_{x}\right)$ $\left(\mathrm{Ti}_{0.95} \mathrm{Zr}_{0.05}\right)_{2.25} \mathrm{O}_{6}$, 当 $x=0.24$ 时样品仍保持透明, 此 时 $\mathrm{Er}^{3+}$ 和 $\mathrm{Yb}^{3+}$ 的总掺杂量达 $30 \%$, 说明 $\mathrm{La}_{2} \mathrm{O}_{3}-\mathrm{TiO}_{2}-\mathrm{ZrO}_{2}$ 基质玻璃具有较高的稀土离子溶解度。这是因为 $\mathrm{La}^{3+}$ 与 $\mathrm{Er}^{3+} 、 \mathrm{Yb}^{3+}$ 同为镧系元素, 电子结构和离子半 径等类似, 有利于在基质玻璃中进行高浓度的稀土 离子掺杂。

\section{2 热学性能分析}

利用差热分析可以获得玻璃的特征温度，从而 衡量玻璃的热稳定性。LTZ: $\mathrm{Er}^{3+} / \mathrm{Yb}^{3+}$ 玻璃的 DTA 曲 线形状大致相同，图 2 是 1\#玻璃的 DTA 曲线, 其玻 璃化转变温度 $T_{\mathrm{g}}=818^{\circ} \mathrm{C}$, 析晶起始温度 $T_{\mathrm{o}}=906^{\circ} \mathrm{C}$ 。而 氟化物或氟氧化物的 $T_{\mathrm{g}}$ 和 $T_{\mathrm{o}}$ 一般均低于 $400^{\circ} \mathrm{C}^{[20]}$ 。 所以, LTZ: $\mathrm{Er}^{3+} / \mathrm{Yb}^{3+}$ 玻璃具有优良的热稳定性，可以 在很宽的温度范围内应用于光学设备中。 $T_{\mathrm{o}}$ 和 $T_{\mathrm{g}}$ 的差 值 $\Delta T\left(\Delta T=T_{0}-T_{\mathrm{g}}\right)$ 常用来表征玻璃的形成能力。 $\Delta T$ 越 大, 玻璃的形成能力越强。1 1 玻璃的 $\Delta T=88^{\circ} \mathrm{C}$, 远低 于硅酸盐或硼酸盐等传统氧化物玻璃 ${ }^{[21-22]}$, 甚至低 于一些重金属氧化物玻璃 ${ }^{[23-24]}$, 可见 $\mathrm{LTZ:} \mathrm{Er}^{3+} / \mathrm{Yb}^{3+}$ 玻璃的形成能力较弱, 难以通过传统的熔融-淬冷 法获得块状玻璃。本实验利用气悬浮技术制备一系 列球状钛酸盐玻璃，表明该技术在制备重金属氧化 物玻璃上具有极大的优势。

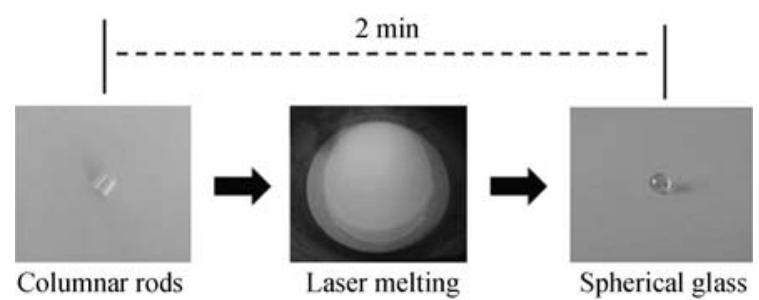

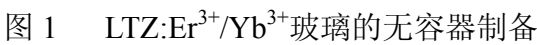

Fig. 1 Containerless processing of LTZ: $\mathrm{Er}^{3+} / \mathrm{Yb}^{3+}$ glasses

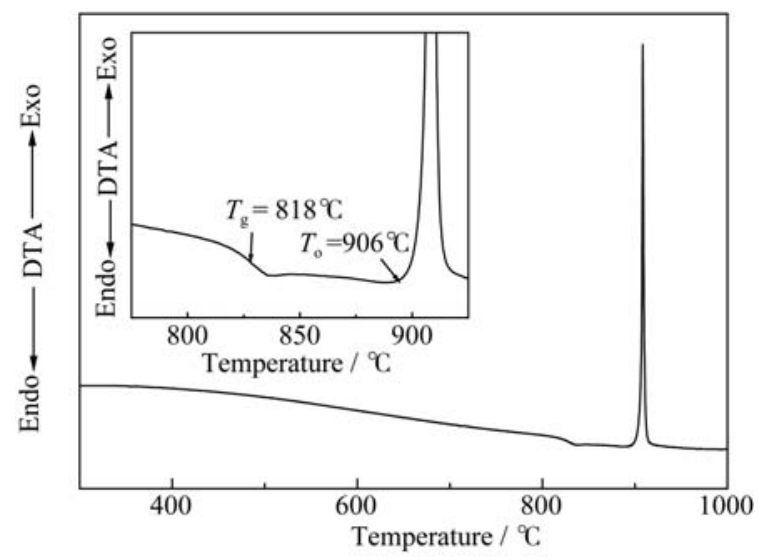

图 21 \#玻璃的 DTA 曲线

Fig. 2 DTA curve of 1 \# glass
图 3 给出了 $T_{\mathrm{g}} 、 T_{\mathrm{o}}$ 和 $\Delta T$ 随 $\mathrm{Yb}^{3+}$ 浓度的变化关 系, 随着 $\mathrm{Yb}^{3+}$ 浓度的不断增加, $T_{\mathrm{g}} 、 T_{\mathrm{o}}$ 和 $\Delta T$ 均呈现 下降趋势, 热稳定性下降, 玻璃的形成能力也降低。 这主要是由于随着稀土元素掺杂量的不断增加, $\mathrm{LTZ}: \mathrm{Er}^{3+} / \mathrm{Yb}^{3+}$ 玻璃中稀土离子会积聚于网络空隙或 部分稀土离子替代网络形成元素进入网络, 使得玻 璃的析晶倾向增强，玻璃形成能力减弱 ${ }^{[25-26]}$ 。

\section{3 吸收/透过光谱}

利用紫外-可见-近红外分光光度计测定了室温 下不同 $\mathrm{Yb}^{3+}$ 浓度的 $\mathrm{LTZ}: \mathrm{Er}^{3+} / \mathrm{Yb}^{3+}$ 玻璃在 400 1600 nm 范围内的吸收曲线，如图 4 所示。10 个吸收峰中心 分别位于 1529、975、797、654、543、522、490、 $452 、 443$ 和 $407 \mathrm{~nm}$ ，对应于基态到激发态 ${ }^{4} \mathrm{I}_{13 / 2}$ 、 ${ }^{4} \mathrm{I}_{11 / 2}\left(\mathrm{Er}^{3+}\right)+{ }^{2} \mathrm{~F}_{5 / 2}\left(\mathrm{Yb}^{3+}\right) 、{ }^{4} \mathrm{I}_{9 / 2} 、{ }^{4} \mathrm{~F}_{9 / 2} 、{ }^{4} \mathrm{~S}_{3 / 2}$ 、 ${ }^{2} \mathrm{H}_{11 / 2} 、{ }^{4} \mathrm{~F}_{7 / 2} 、{ }^{4} \mathrm{~F}_{5 / 2} 、{ }^{4} \mathrm{~F}_{3 / 2}$ 和 ${ }^{2} \mathrm{H}_{9 / 2}$ 能级的跃迁。 未掺入 $\mathrm{Yb}^{3+}$ 时, $975 \mathrm{~nm}$ 处的吸收峰强度较弱; 随着 掺杂 $\mathrm{Yb}^{3+}$ 浓度不断增加, 该吸收峰强度显著增强, 表明 $\mathrm{Yb}^{3+}$ 在 $975 \mathrm{~nm}$ 处的吸收截面远大于 $\mathrm{Er}^{3+}$ 。所 以，采用 $\mathrm{Yb}^{3+}$ 与 $\mathrm{Er}^{3+}$ 共掺，可以增强玻璃对泵浦光

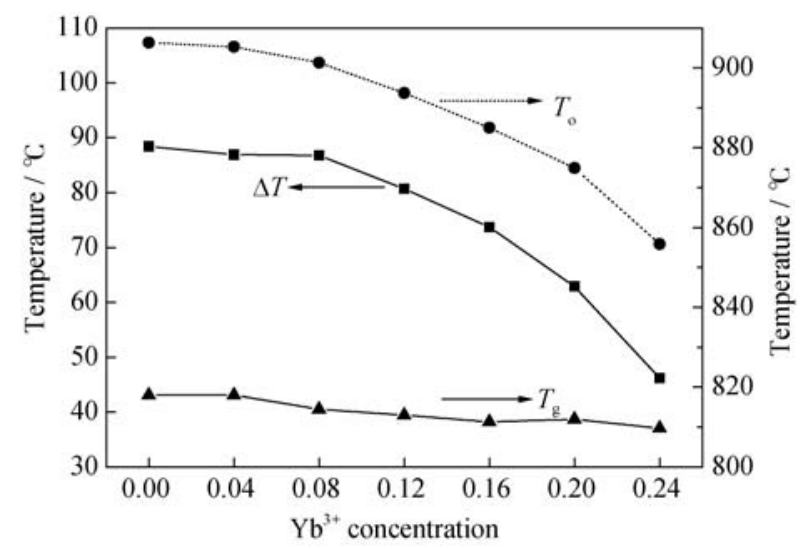

图 $3 \mathrm{LTZ:} \mathrm{Er}^{3+} / \mathrm{Yb}^{3+}$ 玻璃的热稳定性参数与 $\mathrm{Yb}^{3+}$ 浓度的关系 Fig. 3 Dependence of thermal stability parameters of LTZ: $\mathrm{Er}^{3+} / \mathrm{Yb}^{3+}$ glasses on $\mathrm{Yb}^{3+}$ concentration

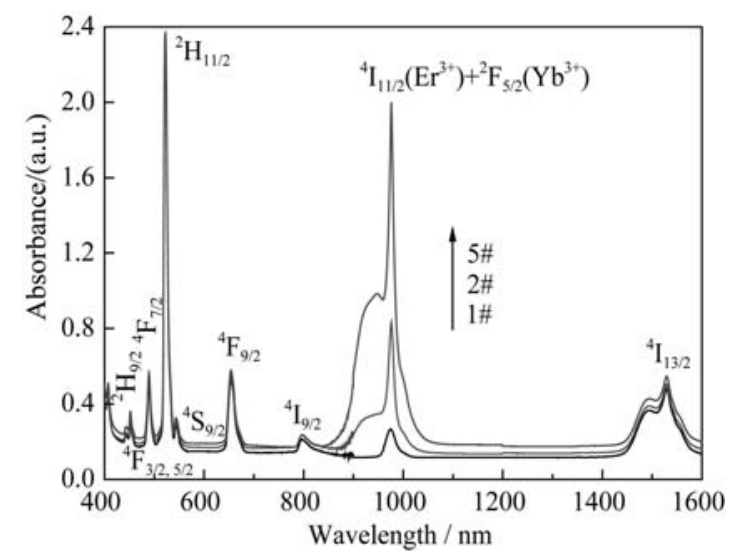

图 4 不同 $\mathrm{Yb}^{3+}$ 浓度的 $\mathrm{LTZ}: \mathrm{Er}^{3+} / \mathrm{Yb}^{3+}$ 玻璃的吸收光谱 Fig. 4 Absorption spectra of LTZ: $\mathrm{Er}^{3+} / \mathrm{Yb}^{3+}$ glasses with different concentrations of $\mathrm{Yb}^{3+}$ 
源的吸收, 有利于获得更强的上转换发光。

图 5 为 1 \#和 $5 \#$ \#玻璃在 $300 \sim 3000 \mathrm{~nm}$ 范围内的 透过光谱。除 $\mathrm{Er}^{3+}$ 和 $\mathrm{Yb}^{3+}$ 的特征吸收外, 样品的透 过率达 $70 \%$ 左右。材料的理论透过率可通过以下公 式计算: 反射系数 $R=(n-1)^{2} /(n+1)^{2}$, 透过率 $T=(1-R)^{2}$ 。按折射率 $n=2.37$ 计算得理论透过率为 $69.7 \%$, 实验结果与理论值十分接近。由于 LTZ: $\mathrm{Er}^{3+} / \mathrm{Yb}^{3+}$ 玻璃在可见光范围具有高透过率, 可 以有效输出上转换发光。

\section{4 上转换发光及其机理}

图 6 为常温下 $980 \mathrm{~nm}$ 泵浦时不同 $\mathrm{Yb}^{3+}$ 浓度的 LTZ: $\mathrm{Er}^{3+} / \mathrm{Yb}^{3+}$ 玻璃的上转换荧光光谱, 它由发光中 心位于 535、554 和 $672 \mathrm{~nm}$ 的三个发光带组成, 分 别对应 $\mathrm{Er}^{3+}$ 的 ${ }^{2} \mathrm{H}_{11 / 2} \rightarrow{ }^{4} \mathrm{I}_{15 / 2}$ 、 ${ }^{4} \mathrm{~S}_{3 / 2} \rightarrow{ }^{4} \mathrm{I}_{15 / 2}$ 和 ${ }^{4} \mathrm{~F}_{9 / 2} \rightarrow{ }^{4} \mathrm{I}_{15 / 2}$ 跃迁 ${ }^{[14]}$ 。图 6 插图显示, 5\#玻璃在 $40.2 \mathrm{~mW}$ 激光葲浦下即可发出肉眼可见的明亮绿 光。图 7 给出了 $535 、 554 \mathrm{~nm}$ 绿光强度和 $672 \mathrm{~nm}$ 红 光强度与 $\mathrm{Yb}^{3+}$ 浓度的关系曲线。随着 $\mathrm{Yb}^{3+}$ 浓度的增

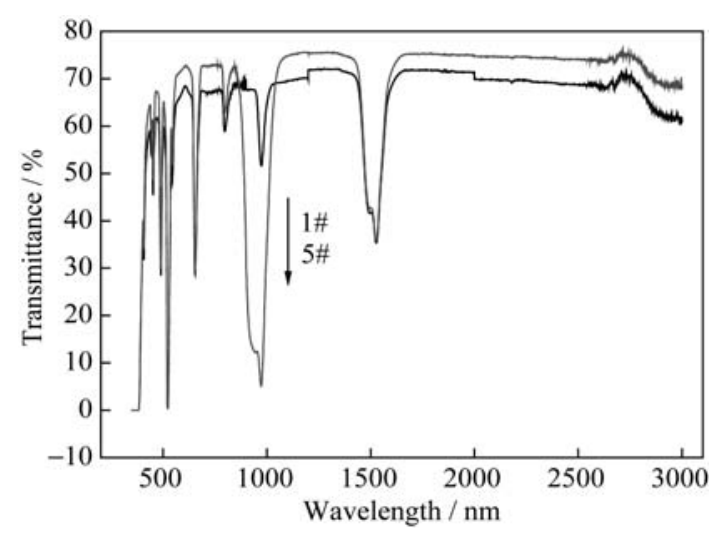

图 5 不同 $\mathrm{Yb}^{3+}$ 浓度的 $\mathrm{LTZ}: \mathrm{Er}^{3+} / \mathrm{Yb}^{3+}$ 玻璃的透过光谱

Fig. 5 Transmission spectra of LTZ: $\mathrm{Er}^{3+} / \mathrm{Yb}^{3+}$ glasses with different concentrations of $\mathrm{Yb}^{3+}$

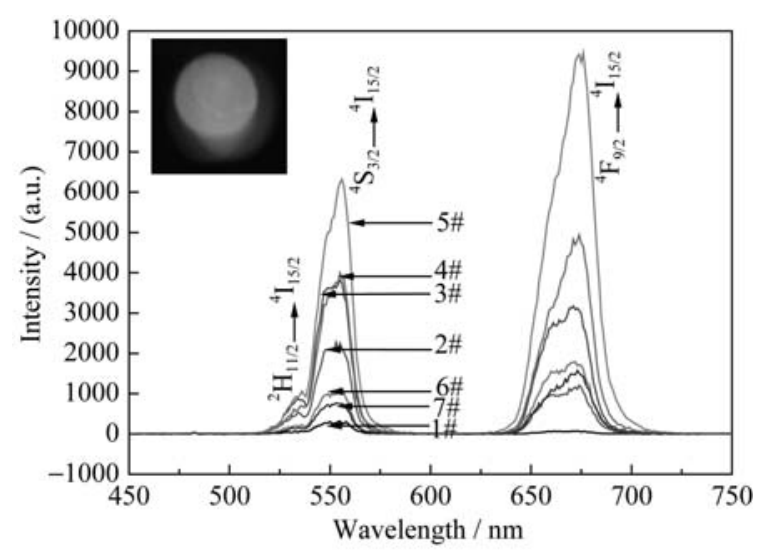

图 6 不同 $\mathrm{Yb}^{3+}$ 浓度的 $\mathrm{LTZ}: \mathrm{Er}^{3+} / \mathrm{Yb}^{3+}$ 玻璃的上转换光谱

Fig. 6 Upconversion emission spectra of $\mathrm{LTZ}: \mathrm{Er}^{3+} / \mathrm{Yb}^{3+}$ glasses with different concentrations of $\mathrm{Yb}^{3+}$

(The insert is the photo of the sample $5 \#$ excited with $40.2 \mathrm{~mW}$ )
加, 绿光和红光强度均呈现先增加后减小的趋势。当 $x=0.16$ 时, $\mathrm{LTZ}: \mathrm{Er}^{3+} / \mathrm{Yb}^{3+}$ 玻璃的上转换发光强度达到 最大值, 此时 $535 \mathrm{~nm}$ 绿光强度是未掺 $\mathrm{Yb}^{3+}$ 时的 13 倍, $554 \mathrm{~nm}$ 绿光强度是未掺 $\mathrm{Yb}^{3+}$ 的 20 倍, $672 \mathrm{~nm}$ 红光 是未掺 $\mathrm{Yb}^{3+}$ 的 130 倍左右。当 $x>0.16$ 后, 上转换发 光强度迅速降低, 这是由于 $\mathrm{Yb}^{3+}$ 浓度过高, 掺杂离 子间相互作用, 发生了浓度猝灭 ${ }^{[27]}$ 。为了解释 $\mathrm{LTZ}: \mathrm{Er}^{3+} / \mathrm{Yb}^{3+}$ 玻璃的上转换发光机理,测量了不同泵 浦功率下的上转换苂光光谱, 分析了发光强度 $I$ 与 泵浦功率 $P$ 的关系。当洜浦功率低于饱和值时, 发 光强度与泵浦功率满足 $I \propto P^{n}$, 式中 $I$ 为上转换发光 强度, $P$ 为百浦功率, $n$ 为发射一个上转换光子所吸 收的泵浦光子数 ${ }^{[28]}$ 。因此, $n$ 为 $\lg I-\lg P$ 曲线的斜率。 通过线性拟合 $\lg I-\lg P$, 得到图 8 。曲线斜率 $n$ 均接近 2, 所以 535、554 nm 处绿光和 $672 \mathrm{~nm}$ 处红光发光均 是双光子机制。

$\mathrm{Er}^{3+} / \mathrm{Yb}^{3+}$ 共掺玻璃的发光机理已有不少报道 ${ }^{[28-31]}$ 。 通过分析 $\mathrm{LTZ}: \mathrm{Er}^{3+} / \mathrm{Yb}^{3+}$ 玻璃的上转换荧光光谱, 结 合稀土离子的能级结构, 阐明了玻璃的上转换发光 机理, 结果如图 9 所示。对于 535 和 $554 \mathrm{~nm}$ 绿光, 上

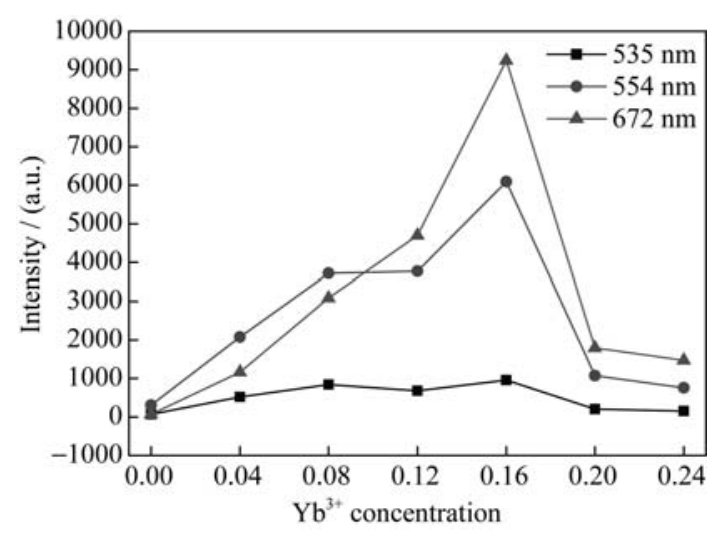

图 $7535,554 \mathrm{~nm}$ 绿光和 $672 \mathrm{~nm}$ 红光强度与 $\mathrm{Yb}^{3+}$ 浓度的关系 Fig. 7 Dependence of luminescence intensities of LTZ: $\mathrm{Er}^{3+}$ / $\mathrm{Yb}^{3+}$ glasses at 535,554 and $672 \mathrm{~nm}$ on $\mathrm{Yb}^{3+}$ concentration

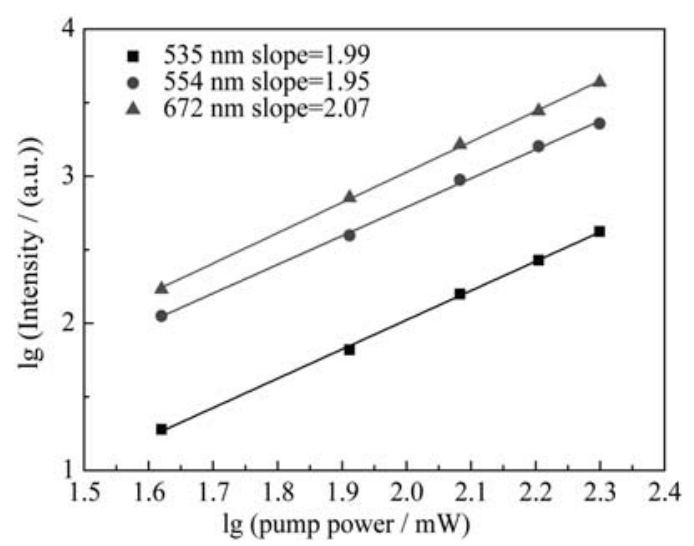

图 $8535 、 554 \mathrm{~nm}$ 绿光和 $672 \mathrm{~nm}$ 红光强度与泵浦功率的关系 Fig. 8 Dependence of luminescence intensities of $\mathrm{LTZ}: \mathrm{Er}^{3+} / \mathrm{Yb}^{3+}$ glasses at 535, 554 and $672 \mathrm{~nm}$ on pump power 
转换过程可能是 $\mathrm{Er}^{3+}$ 通过基态吸收 ${ }^{4} \mathrm{I}_{15 / 2}\left(\mathrm{Er}^{3+}\right)+{ }^{2} \mathrm{~F}_{5 / 2}\left(\mathrm{Yb}^{3+}\right) \rightarrow{ }^{4} \mathrm{I}_{11 / 2}\left(\mathrm{Er}^{3+}\right)+{ }^{2} \mathrm{~F}_{7 / 2}\left(\mathrm{Yb}^{3+}\right)$ $(\mathrm{ET})^{[26]}$ 跃迁至 ${ }^{4} \mathrm{I}_{11 / 2}$ 。 ${ }^{4} \mathrm{I}_{11 / 2}$ 能级上的 $\mathrm{Er}^{3+}$ 通过 ${ }^{4} \mathrm{I}_{11 / 2}\left(\mathrm{Er}^{3+}\right)+{ }^{2} \mathrm{~F}_{5 / 2}\left(\mathrm{Yb}^{3+}\right) \rightarrow{ }^{4} \mathrm{~F}_{7 / 2}\left(\mathrm{Er}^{3+}\right)+{ }^{2} \mathrm{~F}_{7 / 2}\left(\mathrm{Yb}^{3+}\right)$ (ET)、 ${ }^{4} \mathrm{I}_{11 / 2}\left(\mathrm{Er}^{3+}\right)+\mathrm{h} v \rightarrow{ }^{4} \mathrm{~F}_{7 / 2}\left(\mathrm{Er}^{3+}\right)(\mathrm{ESA})$ 跃迁至 ${ }^{4} \mathrm{~F}_{7 / 2}$ 能级。另外，位于 ${ }^{4} \mathrm{I}_{11 / 2}$ 能级的 $\mathrm{Er}^{3+}$ 间还可发生 交叉弛豫 ${ }^{4} \mathrm{I}_{11 / 2}\left(\mathrm{Er}^{3+}\right)+{ }^{4} \mathrm{I}_{11 / 2}\left(\mathrm{Er}^{3+}\right) \rightarrow{ }^{4} \mathrm{~F}_{7 / 2}\left(\mathrm{Er}^{3+}\right)$ $+{ }^{4} \mathrm{I}_{15 / 2}\left(\mathrm{Er}^{3+}\right)$ (CR) 而达到 ${ }^{4} \mathrm{~F}_{7 / 2}$ 能级 ${ }^{[8]}$ 。由于 ${ }^{4} \mathrm{~F}_{7 / 2}$ 能级与 ${ }^{2} \mathrm{H}_{11 / 2} 、{ }^{4} \mathrm{~S}_{3 / 2}$ 能级间的能量差很小, 位于 ${ }^{4} \mathrm{~F}_{7 / 2}$ 能级的 $\mathrm{Er}^{3+}$ 很快无辐射弛豫至 ${ }^{2} \mathrm{H}_{11 / 2} 、{ }^{4} \mathrm{~S}_{3 / 2}$ 能 级。位于 ${ }^{2} \mathrm{H}_{11 / 2}$ 能级的 $\mathrm{Er}^{3+}$ 部分跃迁至 ${ }^{4} \mathrm{I}_{15 / 2}$ 能级, 发出 $535 \mathrm{~nm}$ 绿光; 另一部分则无辐射弛豫至 ${ }^{4} \mathrm{~S}_{3 / 2}$ 能 级, 累积至 ${ }^{4} \mathrm{~S}_{3 / 2}$ 能级的 $\mathrm{Er}^{3+}{ }^{3}$ 跃迁至基态发出 $554 \mathrm{~nm}$ 绿光。因此, 有效的 ET 和 ESA 是绿光发光过程的主 要上转换机制。

由于 ${ }^{2} \mathrm{H}_{11 / 2}$ 和 ${ }^{4} \mathrm{~S}_{3 / 2}$ 能级的能量差很小, 能级间存 在快速的热平衡过程, 可用 $535 \mathrm{~nm}$ 和 $554 \mathrm{~nm}$ 绿光的 强度比来表征 ${ }^{[4,8]}$ 。 $N_{\mathrm{H}} / N_{\mathrm{S}}(\%)=\exp \left(-\Delta E_{\mathrm{g}} / k_{\mathrm{B}} T\right) \times 100$ 表 示两能级上粒子的数目比, 其中 $N_{\mathrm{H}} 、 N_{\mathrm{S}}$ 是 ${ }^{2} \mathrm{H}_{11 / 2}$ 、 ${ }^{4} \mathrm{~S}_{3 / 2}$ 能级上 $\mathrm{Er}^{3+}$ 的数目, $k_{\mathrm{B}}$ 是玻尔兹曼常数, $\Delta E_{\mathrm{g}}$ 是两 能级的能量差, $T$ 为温度。室温下, $N_{\mathrm{H}} / N_{\mathrm{S}}(\%) \approx 2.5 \%$, 所 以从图 6、图 7 中可以看到 $554 \mathrm{~nm}$ 绿光强度远大于 $535 \mathrm{~nm}$ 绿光强度。

$672 \mathrm{~nm}$ 红光是位于 ${ }^{4} \mathrm{~F}_{9 / 2}$ 能级的 $\mathrm{Er}^{3+}$ 辐射跃迁至 基态发出的。 ${ }^{4} \mathrm{~F}_{9 / 2}$ 能级上的 $\mathrm{Er}^{3+}$ 累积过程可能如下: (1)分别由 ${ }^{2} \mathrm{H}_{11 / 2} 、{ }^{4} \mathrm{~S}_{3 / 2}$ 能级无辐射弛豫至 ${ }^{4} \mathrm{~F}_{9 / 2}$ 能级; (2) 由于 ${ }^{4} I_{11 / 2}$ 和 ${ }^{4} I_{13 / 2}$ 能级的能量差很小, ${ }^{4} I_{11 / 2}$ 能级 上的部分 $\mathrm{Er}^{3+}$ 无辐射弛豫至 ${ }^{4} \mathrm{I}_{13 / 2}$, 接着通过

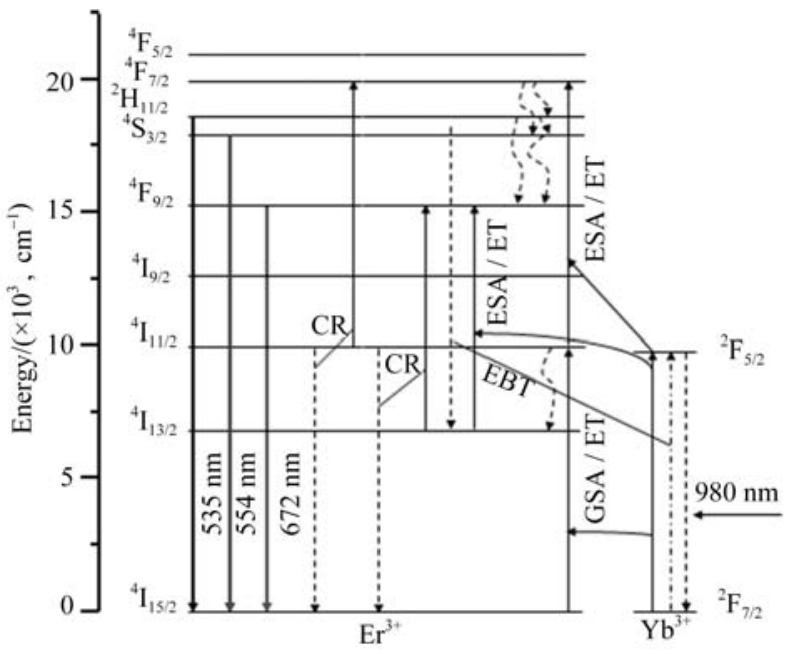

图 $9 \mathrm{LTZ}: \mathrm{Er}^{3+} / \mathrm{Yb}^{3+}$ 玻璃的上转换发光机理简图

Fig. 9 Schematic illustration of the upconversion luminescence of LTZ: $\mathrm{Er}^{3+} / \mathrm{Yb}^{3+}$ glasses
${ }^{4} \mathrm{I}_{13 / 2}\left(\mathrm{Er}^{3+}\right)+{ }^{2} \mathrm{~F}_{5 / 2}\left(\mathrm{Yb}^{3+}\right) \rightarrow{ }^{4} \mathrm{~F}_{9 / 2}\left(\mathrm{Er}^{3+}\right)+{ }^{2} \mathrm{~F}_{7 / 2}\left(\mathrm{Yb}^{3+}\right)$ (ET) 或 ${ }^{4} \mathrm{I}_{13 / 2}\left(\mathrm{Er}^{3+}\right)+\mathrm{h} v \rightarrow{ }^{4} \mathrm{~F}_{9 / 2}\left(\mathrm{Er}^{3+}\right)(\mathrm{ESA})$ 跃迁至 ${ }^{4} \mathrm{~F}_{9 / 2}$ 能级; (3) $\mathrm{Er}^{3+}$ 之间发生交叉弛豫 ${ }^{4} \mathrm{I}_{13 / 2}\left(\mathrm{Er}^{3+}\right)+{ }^{4} \mathrm{I}_{11 / 2}\left(\mathrm{Er}^{3+}\right) \rightarrow{ }^{4} \mathrm{~F}_{9 / 2}\left(\mathrm{Er}^{3+}\right)+{ }^{4} \mathrm{I}_{15 / 2}\left(\mathrm{Er}^{3+}\right)$ $(\mathrm{CR})^{[7]}$, 从而达到 ${ }^{4} \mathrm{~F}_{9 / 2}$ 能级的粒子累积。

从图 7 中可以看出, 当 $\mathrm{Yb}^{3+}$ 的掺杂浓度较低时, $672 \mathrm{~nm}$ 红光强度低于 $554 \mathrm{~nm}$ 绿光; 当 $\mathrm{Yb}^{3+}$ 掺杂浓度 达到 $x=0.12,672 \mathrm{~nm}$ 的红光强度大于 $554 \mathrm{~nm}$ 的绿光 强度。因为 $\mathrm{Yb}^{3+}$ 掺杂浓度的高低不仅会影响样品上 转换发光的整体强度, 还会影响红绿光强度比。当掺 入适量低浓度的 $\mathrm{Yb}^{3+}$ 时, $\mathrm{Yb}^{3+}$ 到 $\mathrm{Er}^{3+}$ 高效的能量转移 使玻璃的上转换绿光和红光强度不断增强。继续提 高 $\mathrm{Yb}^{3+}$ 浓度, 会发生 $\mathrm{Er}^{3+}$ 到 $\mathrm{Yb}^{3+}$ 的能量反向传递 ${ }^{2} \mathrm{H}_{11 / 2} /{ }^{4} \mathrm{~S}_{3 / 2}\left(\mathrm{Er}^{3+}\right)+{ }^{2} \mathrm{~F}_{7 / 2}\left(\mathrm{Yb}^{3+}\right) \rightarrow{ }^{4} \mathrm{I}_{13 / 2}\left(\mathrm{Er}^{3+}\right)+{ }^{2} \mathrm{~F}_{5 / 2}$ $\left(\mathrm{Yb}^{3+}\right)(\mathrm{EBT})$ 过程 ${ }^{[19,31-32]}$ 。因而, ${ }^{4} \mathrm{I}_{13 / 2}$ 能级上的 $\mathrm{Er}^{3+}$ 数量增加, 可通过 ESA 或 ET 过程进一步累积到 ${ }^{4} \mathrm{~F}_{9 / 2}$ 能级, 跃迁至基态发出红光, 使得红光强度大 大增强。此外, 由于 ${ }^{4} \mathrm{I}_{13 / 2}$ 能级上的 $\mathrm{Er}^{3+}$ 数量增加, $\left({ }^{4} \mathrm{I}_{13 / 2},{ }^{4} \mathrm{I}_{11 / 2}\right) \rightarrow\left({ }^{4} \mathrm{I}_{15 / 2},{ }^{4} \mathrm{~F}_{9 / 2}\right)$ 过程也会增强, 使红光 强度增加。同时 EBT 过程使得 ${ }^{2} \mathrm{H}_{11 / 2} 、{ }^{4} \mathrm{~S}_{3 / 2}$ 能级上 的 $\mathrm{Er}^{3+}$ 数目有一定程度的减少, 这与 $x=0.12$ 时 $672 \mathrm{~nm}$ 红光强度的增加幅度大于 535、554 nm 绿光吻合(图 $7)$ 。当 $\mathrm{Yb}^{3+}$ 掺杂浓度 $x>0.16$, 发生浓度猝灭, 535、554 和 $672 \mathrm{~nm}$ 处的发光强度都迅速减弱。这是由于玻璃 中掺杂稀土离子浓度过高, $\mathrm{Er}^{3+}$ 和 $\mathrm{Yb}^{3+}$ 间及 $\mathrm{Yb}^{3+}$ 相互 之间距离较近, 激发能在 $\mathrm{Er}^{3+}$ 和 $\mathrm{Yb}^{3+}$ 间不断传递而 损失, 导致绿光和红光强度均降低。因此, EBT、ESA 及 ET 双光子过程是红光发光过程的主要机制。

\section{3 结论}

1) 利用气悬浮技术成功地获得了不同稀土离 子掺杂浓度的透明球状 LTZ: $\mathrm{Er}^{3+} / \mathrm{Yb}^{3+}$ 玻璃, 稀土离 子的掺杂浓度最高达到 $30 \%$ 。

2) $\mathrm{LTZ}: \mathrm{Er}^{3+} / \mathrm{Yb}^{3+}$ 玻璃具有良好的热稳定性, 但其 热稳定性和玻璃形成能力随 $\mathrm{Yb}^{3+}$ 浓度的增加而降低。

3) $\mathrm{Yb}^{3+}$ 与 $\mathrm{Er}^{3+}$ 共掺可以提高 $\mathrm{LTZ}: \mathrm{Er}^{3+} / \mathrm{Yb}^{3+}$ 玻璃 对 $980 \mathrm{~nm}$ 百浦光的吸收, 且在可见光范围内透光率 达到 $70 \%$ 左右, 可以有效输出上转换发光。

4) 随着 $\mathrm{Yb}^{3+}$ 浓度的增加, LTZ: $\mathrm{Er}^{3+} / \mathrm{Yb}^{3+}$ 玻璃的 上转换发光强度先增大后减小。 $\mathrm{Yb}^{3+}$ 对于 $\mathrm{Er}^{3+}$ 有很 强的敏化作用，且对于红光的影响强于绿光，当 $\mathrm{Yb}^{3+}$ 掺入浓度 $x=0.16$ 时, 上转换发光强度达到最大 
值。 $\mathrm{LTZ}: \mathrm{Er}^{3+} / \mathrm{Yb}^{3+}$ 玻璃主要的上转换机制是 $\mathrm{ET}$ 、 $\mathrm{ESA}$ 及 $\mathrm{EBT}$ 双光子过程。

\section{参考文献:}

[1] QIAO Y B, CHEN D P, REN J J, et al. Blue emission from $\mathrm{Eu}^{2+}$-doped high silica glass by near-infrared femtosecond laser irradiation. J. Appl. Phys., 2008, 103(2): 023108-1-3.

[2] CAMILO M E, ASSUMPÇÃO T A A, DA SILVA D M, et al. Influence of silver nanoparticles on the infrared-to-visible frequency upconversion in $\mathrm{Tm}^{3+} / \mathrm{Er}^{3+} / \mathrm{Yb}^{3+}$ doped $\mathrm{GeO}_{2}-\mathrm{PbO}$ glass. J. Appl. Phys., 2013, 113(15): 153507-1-4.

[3] MIGUEL A, MOREA R, GONZALO J, et al. Near-infrared emission and upconversion in $\mathrm{Er}^{3+}$-doped $\mathrm{TeO}_{2}-\mathrm{ZnO}-\mathrm{ZnF}_{2}$ glasses. $J$. Lumin., 2013, 140: 38-44.

[4] SOM T, KARMAKAR B. Efficient green and red fluorescence upconversion in erbium doped new low phonon antimony glasses. Opt. Mater., 2009, 31(4): 609-618.

[5] CRUZ G K, KÖPP F M, VIATROSKI M A, et al. Re-absorption process in the upconversion green emission of the erbium ion-doped fluorozirconate glass system. J. Lumin., 2010, 130(4): 645-647.

[6] HONG J, NIE Q H, DAI S X, et al. Effects of Temperature and ytterbium sensitizing on upconversion characteristics in Er-doped tellurite glasses. Journal of Inorganic Materials, 2008, 23(5): 1033-1036.

[7] MAHESHVARAN K, ARUNKUMAR S, SUDARSAN V, et al. Structural and luminescence studies on $\mathrm{Er}^{3+} / \mathrm{Yb}^{3+}$ co-doped boro-tellurite glasses. J. Alloys Compd., 2013, 561: 142-150.

[8] FENG L, LAI B Y, WANG J, et al. Spectroscopic properties of $\mathrm{Er}^{3+}$ in a oxyfluoride glass and upconversion and temperature sensor behavior of $\mathrm{Er}^{3+} / \mathrm{Yb}^{3+}$-codoped oxyfluoride glass. J. Lumin., 2010, 130(12): 2418-2423.

[9] CHEN X B, SONG Z F, SAWANOBORI N, et al. The characteristic saturation phenomenon of upconversion luminescence in holmiumytterbium-co-doped oxyfluoride glass $\mathrm{Ho}(0.1) \mathrm{Yb}(5)$ :FOG. Phys. B, 2008, 403(21/22): 3847-3852.

[10] MACIEL G S, DEARAUJO C B, MESSADDEQ Y, et al. Frequency upconversion in $\mathrm{Er}^{3+}$-doped fluoroindate glasses pumped at 1.48 um. Phys. Rev. B, 1997, 55(10): 6335-6341.

[11] YANG D L, HOU Y Y, ZHAO X, et al. Intense blue upconversion fluorescence of $\mathrm{Tm}^{3+} / \mathrm{Yb}^{3+}$-codoped bismuth tellurite glasses. Acta Physinica Sinica, 2006, 55(8): 4304-4308.

[12] QIN Z X, YUAN X, XIONG B X, et al. Judd-Ofelt theory analysis and spectroscopic properties of $\mathrm{Nd}^{3+}$-doped $\mathrm{TeO}_{2}-\mathrm{Al}_{2} \mathrm{O}_{3}-\mathrm{Cs}_{2} \mathrm{O}$ tellurite glasses. Acta Photonica Sinica, 2013, 42(1): 1-6.

[13] ZARZYCKI J. X-ray diffraction study of alkali titanate glasses. $J$. Mater. Sci., 1971, 6: 130-135.

[14] YU J D, KOHARA S, ITOH K, et al. Comprehensive structural study of glassy and metastable crystalline $\mathrm{BaTi}_{2} \mathrm{O}_{5}$. Chem. Mater., 2009, 21(2): 259-263.

[15] YU J D, ARAI Y, MASAKI T, et al. Fabrication of $\mathrm{BaTi}_{2} \mathrm{O}_{5}$ glass-ceramics with unusual dielectric properties during crystallization. Chem. Mater., 2006, 18(8): 2169-2173.

[16] INOUE H, WATANABE Y, MASUNO A, et al. Effect of substi- tuting $\mathrm{Al}_{2} \mathrm{O}_{3}$ and $\mathrm{ZrO}_{2}$ on thermal and optical properties of high refractive index $\mathrm{La}_{2} \mathrm{O}_{3}-\mathrm{TiO}_{2}$ glass system prepared by containerless processing. Opt. Mater., 2011, 33(12): 1853-1857.

[17] PAN X H, YU J D, LIU Y, et al. Thermal, mechanical, and upconversion properties of $\mathrm{Er}^{3+} / \mathrm{Yb}^{3+}$ co-doped titanate glass prepared by levitation method. J. Alloys Compd., 2011, 509(27): 7504-7507.

[18] ZHANG M H, LIU Y, YU J D, et al. A novel upconversion $\mathrm{TiO}_{2}-\mathrm{La}_{2} \mathrm{O}_{3}-\mathrm{Ta}_{2} \mathrm{O}_{5}$ bulk glass co-doped with $\mathrm{Er}^{3+} / \mathrm{Yb}^{3+}$ fabricated by containerless processing. Mater. Lett., 2012, 66(1): 367-369.

[19] ZHANG M H, YU J D, PAN X H, et al. Bifunction in $\mathrm{Er}^{3+} / \mathrm{Yb}^{3+}$ co-doped $\mathrm{BaTi}_{2} \mathrm{O}_{5}-\mathrm{Gd}_{2} \mathrm{O}_{3}$ glasses prepared by aerodynamic levitation method. Mater. Res. Bull., 2013, 48(11): 4729-4732.

[20] BABU P, SEO H J, KESAVULU C R, et al. Thermal and optical properties of $\mathrm{Er}^{3+}$-doped oxyfluorotellurite glasses. J. Lumin., 2009, 129(5): 441-448.

[21] ABDEL-HAMEED S A M, EL-KHESHEN A A. Thermal and chemical properties of diopside-wollastonite glass-ceramics in the $\mathrm{SiO}_{2}-\mathrm{CaO}-\mathrm{MgO}$ system from raw materials. Ceram. Int., 2003, 29(3): 265-269.

[22] YANG Y M, YANG Z P, CHEN B J, et al. Spectroscopic properties and thermal stability of $\mathrm{Er}^{3+}$-doped germinate-borate glasses. $J$. Alloys Compd., 2009, 479(1/2): 883-887.

[23] XU T F, ZHANG X D, DAI S X, et al. Effect of $\mathrm{SiO}_{2}$ content on the thermal stability and spectroscopic properties of $\mathrm{Er}^{3+} / \mathrm{Yb}^{3+}$ co-doped tellurite borate glasses. Phys. B, 2007, 389(2): 207-380.

[24] SIMON S, SIMON V. Thermal characterization of gallium-bismuthate oxide glasses. Mater. Lett., 2004, 58(29): 3778-3781.

[25] ZHU J Q, TANG G, BAI C Y, et al. Effect of $\mathrm{Eu}^{3+}, \mathrm{Lu}^{3+}$ on formation of galliom fluoride glasses. Journal of the Chinese Ceramic Society, 2006, 34(3): 353-357.

[26] NI Y R, LU C H, ZHANG Y, et al. Thermal stability of boroaluminosilicate glass with rare earth doping. The Chinese Journal of Process Engineering, 2006, 6(5): 777-780.

[27] CHEN Z S, GONG W P, CHEN T F, et al. Preparation and upconversion luminescence of $\mathrm{Er}^{3+} / \mathrm{Yb}^{3+}$ codoped $\mathrm{Y}_{2} \mathrm{Ti}_{2} \mathrm{O}_{7}$ nanocrystals. Mater. Lett., 2012, 68: 137-139.

[28] VETRONE F, BOYER J C, CAPOBIANCO J A, et al. Significance of $\mathrm{Yb}^{3+}$ concentration on the upconversion mechanisms in codoped $\mathrm{Y}_{2} \mathrm{O}_{3}: \mathrm{Er}^{3+}, \mathrm{Yb}^{3+}$ nanocrystals. J. Appl. Phys., 2004, 96(1): 661-667.

[29] ZHANG G, SONG F, MING C G, et al. Photoluminescence properties and pump-saturation effect of $\mathrm{Er}^{3+} / \mathrm{Yb}^{3+}$ co-doped $\mathrm{Y}_{2} \mathrm{Ti}_{2} \mathrm{O}_{7}$ nanocrystals. J. Lumin., 2012, 132(3): 774-779.

[30] CHENG G M, FENG S, YIN Y, et al. Optical character of $\mathrm{Er}^{3+} / \mathrm{Yb}^{3+}$ co-doped $\mathrm{P}_{2} \mathrm{O}_{5}-\mathrm{CaO}-\mathrm{Na}_{2} \mathrm{O}-\mathrm{Al}_{2} \mathrm{O}_{3}-\mathrm{AgO}$ phosphate glass. Opt. Commun., 2011, 284(7): 1868-1871.

[31] SOLIS D, ROSA L D E, MEZA O, et al. Role of $\mathrm{Yb}^{3+}$ a nd $\mathrm{Er}^{3+}$ concentration on the tunability of green-yellow-red upconversion emission of codoped $\mathrm{ZrO}_{2}: \mathrm{Yb}^{3+}-\mathrm{Er}^{3+}$ nanocrystals. J. Appl. Phys., 2010, 108(2): 023103-1-9.

[32] SONG H W, SUN B J, WANG T, et al. Three-photon upconversion luminescence phenomenon for the green levels in $\mathrm{Er}^{3+}$ $\mathrm{Yb}^{3+}$ codoped cubic nanocrystalline yttria. Solid State Commun., 2004, 132(6): 409-413. 